\title{
WS2-B03
}

\section{Fast Track Broadband Seismic Inversion Workflows}

\author{
A. Geisslinger (Brunei Shell Petroleum Company) \& H.H. Hamzah* (Brunei \\ Shell Petroleum Company)
}

\section{SUMMARY}

This talk will focus on how Fast Track Reservoir Characterization approaches based on broadband data are being applied on a regional seismic data set of $7000 \mathrm{~km} 2$ delivering Calibrated Impedance and Fluid Cubes for the entire regional volume, thus enabling Exploration and Development teams to readily harvest value of the improved rock property characterization through broadband seismic.

1 - Calibrated Impedance

The much improved low frequency content of the broadband data together with higher frequency content of the velocity model generated by modern processing techniques allows us to close the conventional frequency gap at around 1-8 Hz. A seismic velocity model based low frequency P-Impedance model and reflectivity derived bandlimited impedance cube can be merged seamlessly into a calibrated impedance volume.

2 - Fluid Cube

Using Broadband Seismic Data and an operator based Coloured Inversion workflow, followed by weighted stacking of the results, fluid cubes can be generated in a very efficient way. This allows their usage in prospect identification timely after end of a processing project and very early in the exploration interpretation workflows. Having fluid cubes early, is of particular value in the geology of Brunei Darussalam, where prospects are distributed over thousands of milliseconds of sand-shale sequences. 


\section{Amsterdam '14}

\section{Introduction}

This talk will outline how Fast Track Reservoir Characterization approaches based on broadband data are being applied on a seismic data set of $7000 \mathrm{~km} 2$ delivering Calibrated Impedance and a Fluid Cube for the entire regional volume, thus enabling Exploration and Development teams to readily harvest value of the improved rock property characterization through broadband seismic data.

For a long time the main purpose of acquiring, processing and interpreting seismic data was to gain an understanding of the structure of the subsurface. The onset of AVO interpretation lead to the concepts of True Amplitude Processing which enabled rock property derivation through seismic inversions. Practising these workflows made people realize the shortcomings of conventional seismic and the value of the low frequency spectrum got in the focus of the Geophysicist community. More recently Broadband Seismic Data acquisition and processing techniques developed. While part of the community is further improving the acquisition and processing techniques some of us are working on early Broadband Data sets, fine-tuning workflows to derive rock properties.

\section{Fast Track Broadband Seismic Inversion Workflows}

\section{1 - Calibrated Impedance}

The much improved low frequency content of the broadband data (Soubaras, 2010) together with higher frequency content of the velocity model allows us to close the conventional frequency gap at around 1-8 Hz. The bandlimited impedance was derived through a coloured inversion (Lancaster\&Whitcombe, 2000) workflow from a conditioned reflectivity cube. Figure 1 indicates how a seismic velocity model based low frequency P-Impedance volume and the bandlimited impedance cube can be merged seamlessly into a calibrated impedance. This volume was found to have a high fidelity away from well control and can be used to generate rock property volumes, Figure 1.

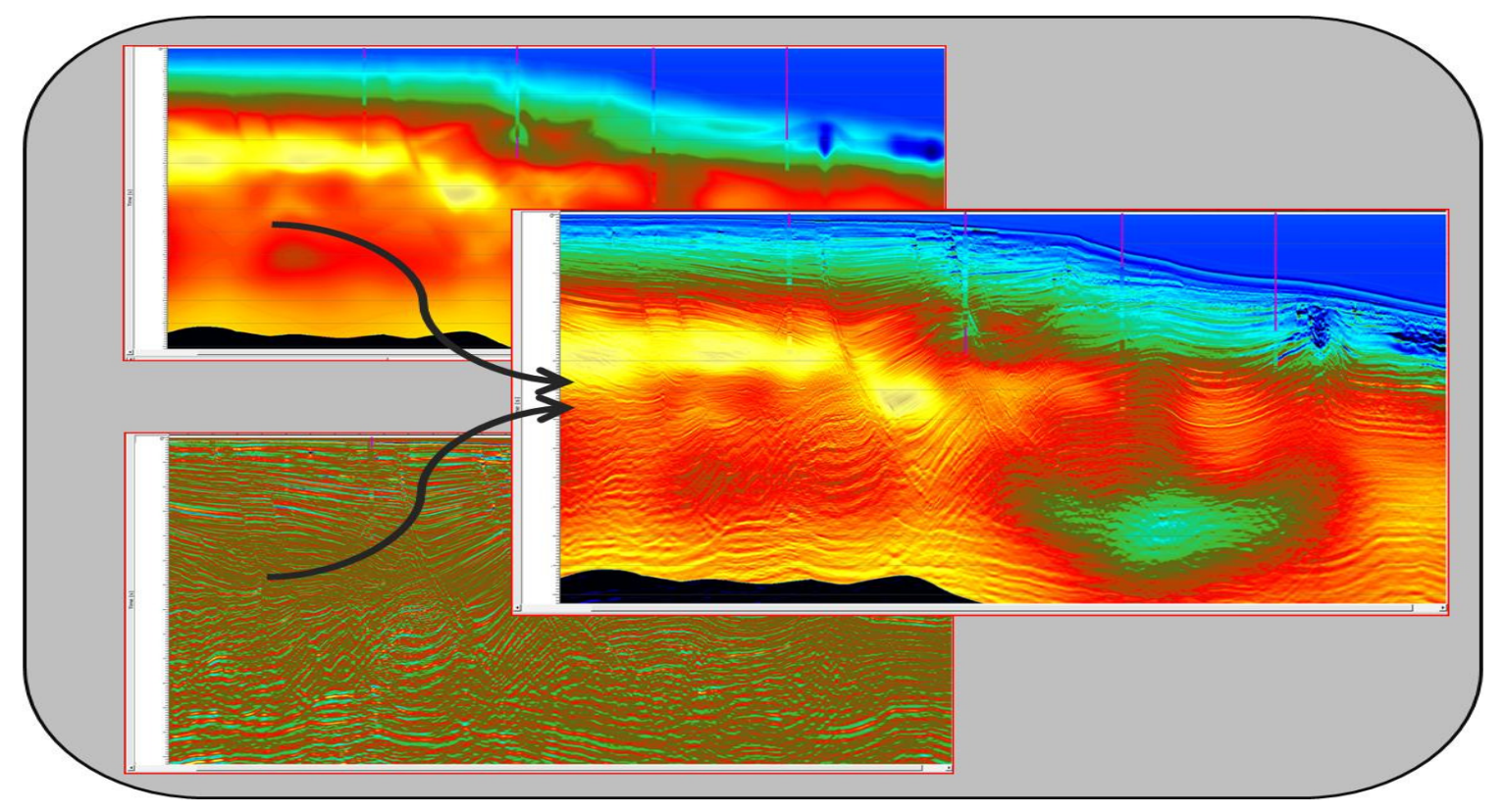

Figure 1 Velocity model derived low frequency model (upper left) is merged with seismically derived bandlimited impedance (lower left) into the final calibrated impedance cube (right). 


\section{Amsterdam ' 14}

\section{2 - Fluid Cube}

Fluid cubes are typically the result of time consuming Quantitative Interpretation workflows and often are generated only for small fractions of the available seismic data. Using Broadband Seismic Data and an operator based Coloured Inversion (Lancaster\&Whitcombe, 2000) workflow, followed by weighted stacking of the results, fluid cubes can be generated in a very efficient way for entire regional seismic data sets. This allows their usage in prospect identification timely after end of a processing project and early in the exploration interpretation workflows. Having fluid cubes early is of particular value in the geology of Brunei Darussalam, where prospects are distributed over thousands of milliseconds of sand-shale sequences. The fluid cube can be calibrated to saturation logs at well locations or DHI's in the seismic data itself. Figure 2 shows an example of an anomaly which was easily identified in the fluid cube, but is barely visible in the full stack seismic data.

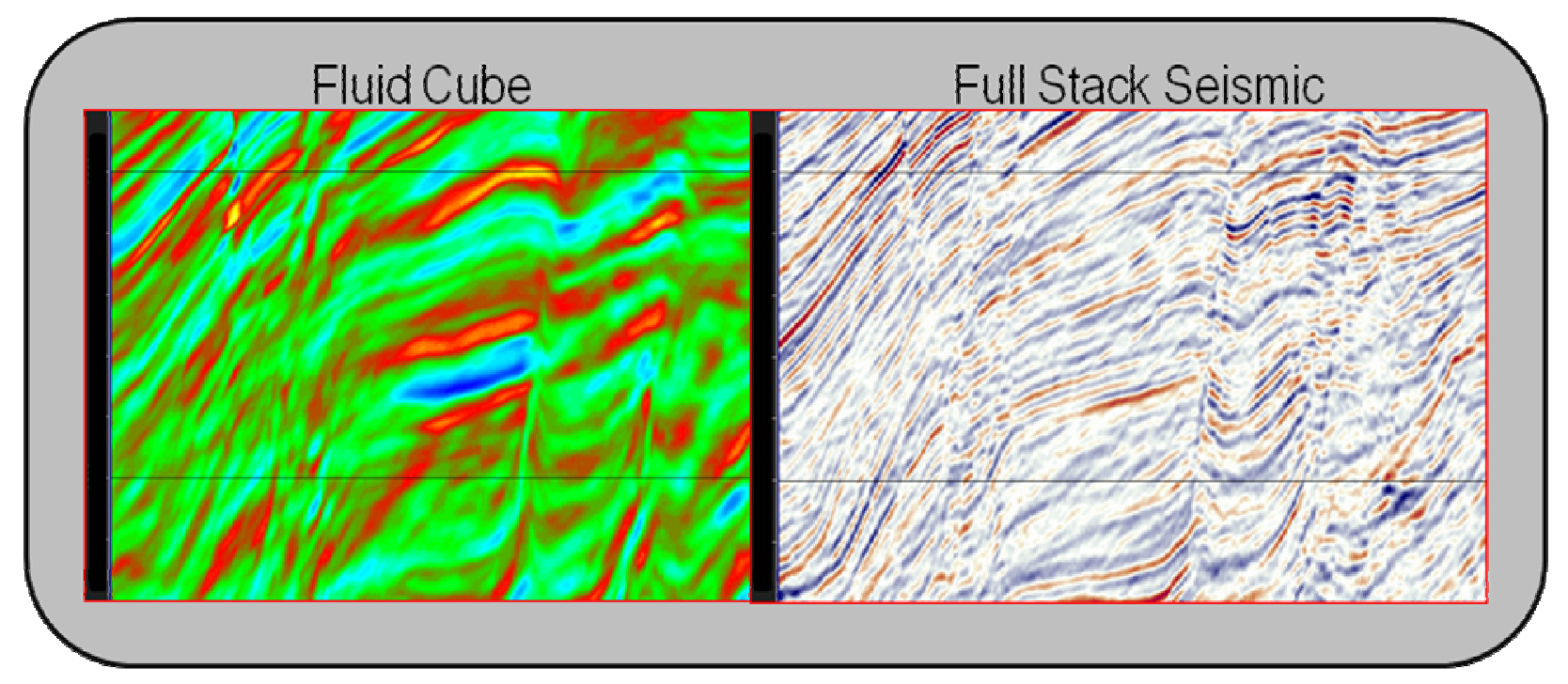

Figure 2 zooms-in on a fluid anomaly (dark blue) which is terminating with flat spot and conformity to structure. In the full stack seismic the related amplitudes are rather undifferentiated and the DHI does not stand out clearly.

\section{Conclusions}

Due to an improved low frequency spectrum the Broadband Seismic Data provides the means to derive rock properties more efficiently, thus making rock property information available more readily particularly for large regional broadband surveys. This has proven a high impact on prospect identification and also cuts down modelling time for seismically constraint reservoir models for identified prospects.

\section{Acknowledgements}

We would like to acknowledge our colleagues in the Brunei Shell Petroleum Geophysics and Exploration departments.

\section{References}

Lancaster, S. and Whitcombe, D. [2000] Fast-track 'coloured' inversion. SEG Expanded Abstracts.

Soubaras, R. and Dowle, R. [2010] Variable-depth streamer - a broadband marine solution. First Break, 28 (12), p. 89-96. 